\section{T. NAIDJI, S. LAMBERT \\ Keller Fondations Spéciales}

\section{P. BERTHELOT}

Bureau Veritas

\title{
Amélioration de sols limoneux vasards par colonnes ballastées
}

et préchargement statique

Sous une première couche de tourbe d'un mètre d'épaisseur, des limons vasards à passées sableuses ont été détectés sur une profondeur de plus de 30 mètres sur le site de construction d'un hypermarché à Ljubljana (Slovénie). Cet environnement géologique particulièrement hostile nous a conduits à réaliser, après purge de la tourbe et mise en place d'une couche drainante, un renforcement homogénéisé de sol par colonnes ballastées, de $18 \mathrm{~m}$ de profondeur $(57000 \mathrm{ml}$ au total), à la place d'une solution par pieux. La surface ainsi traitée a ensuite été préchargée par environ $200000 \mathrm{~m}^{3}$ de remblai. Le suivi tassométrique précis, après mise en place des colonnes de production et pendant les phases de remblaiement et de construction de l'ouvrage, a permis d'affiner en fonction de l'hétérogénéité localisée du sol, la hauteur et la durée du préchargement. Toutes ces observations illustrent parfaitement le comportement d'un sol compressible renforcé par des colonnes ballastées au cours du temps. Elles mettent aussi en évidence la stabilisation des tassements après déchargement.

Mots-clés : colonnes ballastées, suivis tassométriques, préchargement, purge.

\section{Soil improvement of silty clays by stone columns with additional static preloading}

Underneath a one meter thick layer of peat, sandy silts have been found to depths of more than $30 \mathrm{~m}$ on a construction site for a hypermarket at Ljubljana (Slovenia). This hostile geological environment lead us to a solution to reinforce homogeneously the soil by stone colums to a depth of $18 \mathrm{~m}$ (total 57,000 linear meters) after soil substitution of the peat by a draining gravel layer, instead of piling. The entire surface was then preloaded by about $200,000 \mathrm{~m}^{3}$ of earthfill. The follow up of settlements during construction phase and during the phases of preloading have allowed to adjust preloading time and height of the fill in accordance with local variations of the soil. All these observations have allowed to demonstrate perfecty the settlement-time-behaviour of a reinforced compressible soil by stone columns. It demonstrated as well the perfect stabilisation of settlements after the unloading phase.

Key words: stone columns, follow up of settlements, preloading, substitution. 
.8. 1.7

\section{Introduction}

Le site de construction d'un hypermarché à Ljubljana (Slovénie) se caractérise par des conditions géologiques particulièrement difficiles : sous une première couche de tourbe, des limons vasards à passées sableuses ont été détectés sur une profondeur de plus de 30 mètres.



Haune: 1 Photo du bâtiment achevé.

Vis-à-vis d'une solution ( pieux » difficilement envisageable, il a été retenu un renforcement de sol par colonnes ballastées sur 18 mètres de profondeur. L'objectif de ce traitement était de permettre la réalisation du bâtiment en mode superficiel par des fondations isolées et filantes et un dallage sur terre-plein classique. Sous le parking $(4 a, 4 b, 3 a, 3 b, 3 c)$ et la cour de service (4c), une solution de préchargement avec mise en place de drains plats verticaux a été choisie (Fig. 2). Son étude ne fait pas partie de cet article. Le traitement de sol par colonnes ballastées a permis d'augmenter la capacité portante du sol, de diminuer sa compressibilité, d'accélérer les tassements par effet drainant et de réduire les tassements différentiels. Sous le bâtiment, après purge de la tourbe et mise en place d'une couche drainante, des colonnes ballastées ont été réalisées

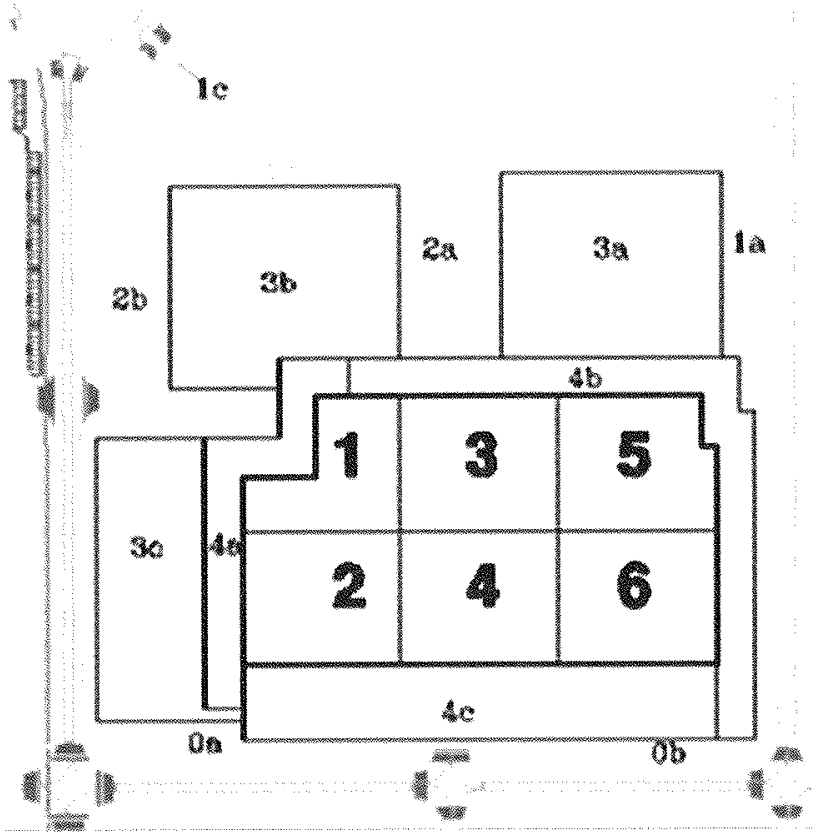

Ћ1G. 2 Vue d'ensemble du site et découpage du bâtiment en zones. suivant un maillage de $3 \times 3 \mathrm{~m}$, à une profondeur de 18 mètres. Compte tenu de la compressibilité du sol, la surface ainsi traitée a été préchargée par un remblai de $1,5 \mathrm{~m}$ de hauteur au-dessus de la cote finie dallage (après réalisation d'un remblai de mise à niveau). Un système de suivi de tassements et de pressions interstitielles a été mis en place avec également la réalisation de 3 plots d'essais de préchargement dans 3 zones différentes.

\section{9}

\section{Données géotechniques}

Les deux campagnes de reconnaissance ont mis en évidence sur plus de 30 mètres d'épaisseur un complexe alluvionnaire constitué essentiellement par :

- des remblais caillouteux compacts sur une épaisseur de 0,80 à $2,00 \mathrm{~m}$;

- du limon tourbeux ou de la tourbe sur une épaisseur de 0,30 à $1,00 \mathrm{~m}$;

- une alternance de passages limoneux et argileux caractérisée par des valeurs de résistance à la pointe qc au CPT de l'ordre de $0,5 \mathrm{MPa}$;

- des limons plus sableux juscu'à 20 m de profondeur caractérisés par des qc variant entre 1 et $2 \mathrm{MPa}$;

- du sable compact jusqu'à 22,50 m ;

et, enfin, à nouveau des limons et argiles molles jusqu'à la base des sondages.

Très peu d'essais de laboratoire étaient à notre disposition en phase d'appel d'offres et une campagne de reconnaissance complémentaire a été lancée pour prélever des échantillons afin de connaître la nature exacte de ces matériaux, leur perméabilité et leur caractère évolutif. Les essais de laboratoire sur les silts prélevés entre 6 et $14 \mathrm{~m}$ ont mis en évidence les caractéristiques suivantes:

- un passant de $80 \mathrm{~m}$, compris entre 92 et $99 \%$, avec une fraction argileuse $(2 \mathrm{~m})$, de l'ordre de $10 \%$;

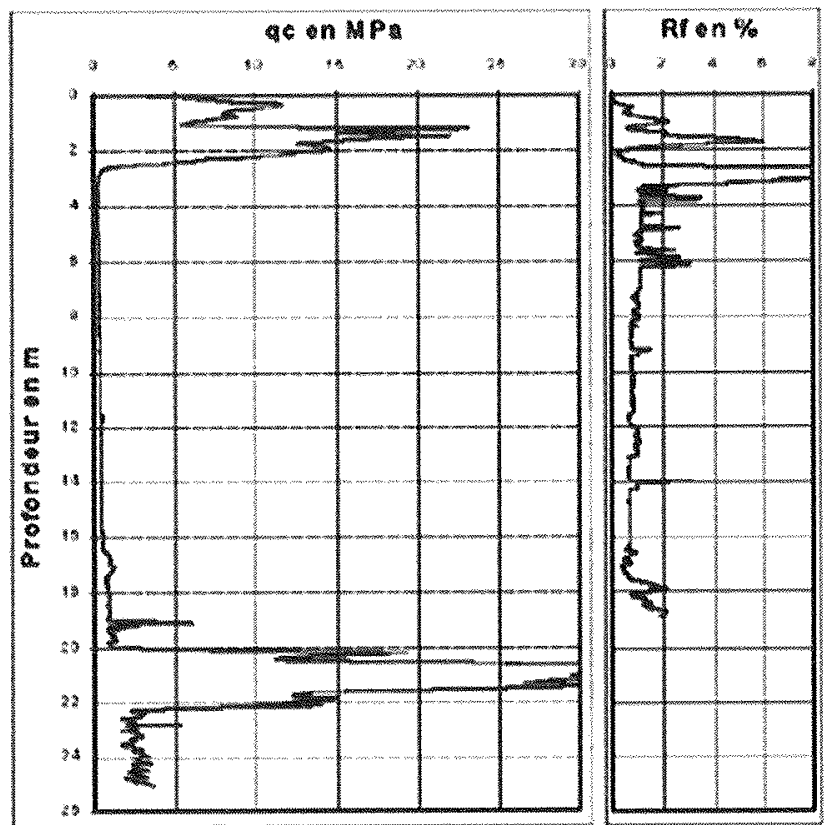

fic. 3 Essai au pénétromètre statique. 
- des teneurs en eau élevées : $32,9 \leq w \leq 98 \%$;

- des valeurs au bleu de méthylène comprises entre 0,8 et 1,5 caractérisant un matériau à fraction argileuse faible ;

- une perméabilité mesurée relativement faible : $1,2 \cdot 10^{-9} \leq \mathrm{k} \leq 3,5 \cdot 10^{-9} \mathrm{~m} / \mathrm{s}$. La perméabilité moyenne de $2.10^{-9} \mathrm{~m} / \mathrm{s}$ a été retenue pour les calculs.

\section{3}

\section{Le projet}

\section{3.}

\section{Généralités}

Le projet comporte un bâtiment commercial de $20000 \mathrm{~m}^{2}$ avec une cour de service de $5700 \mathrm{~m}^{2}$ et $50000 \mathrm{~m}^{2}$ de parking et de voirie. La structure du bâtiment est de type métallicue et les surcharges d'exploitation sur le dallage sont de l'ordre de $20 \mathrm{kN} / \mathrm{m}^{2}$ maximum dans les réserves. Le site se situe dans une zone inondable et la cote du bâtiment et parking a été calée entre 0,50 et $2,10 \mathrm{~m}$ au-dessus du terrain naturel par un remblai de mise à niveau (Fig. 4).

\section{s.}

\section{Traitement sous le bâtiment}

La solution retenue a consisté à réaliser près de $57000 \mathrm{ml}$ de colonnes ballastées selon une maille carrée de $3 \times 3 \mathrm{~m}$ avec une concentration des colonnes sous les massifs de structure. Afin de maîtriser parfaitement les tassements, ce traitement est complété par :

- une purge des matériaux tourbeux de surface ;

- un préchargement par du remblai correspondant à environ 1,5 fois le poids du bâtiment.

L'objectif de ce préchargement était de provoquer un tassement égal ou supérieur à celui qui aurait lieu sous le poids du futur ouvrage; ce qui permettrait de garantir une stabilité définitive du bâtiment à très long terme. Pour le remblaiement de mise à niveau et de préchargement, le bâtiment a été divisé en 6 par-

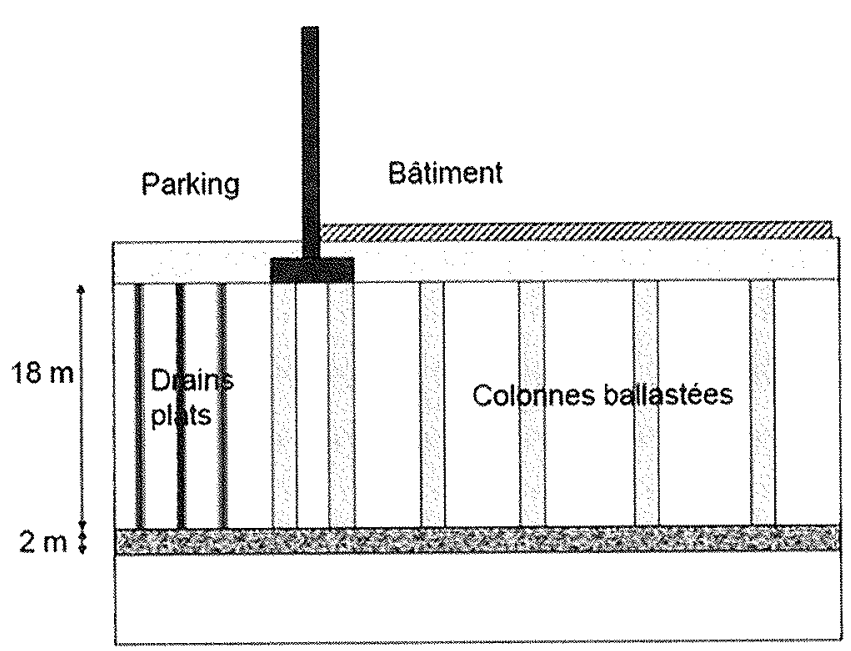

IIC 4 Coupe schématique du traitement de sol. ties fictives. Chaque partie était remblayée jusqu'à sa cote finale de mise à niveau plus $1,50 \mathrm{~m}$ de remblai de surcharge, l'avancement des travaux de terrassement étant réalisé de la zone 1 et 2 vers la zone 5 et 6 (Fig. 4).

\section{5.}

\section{Temps de consolidation}

Le réseau de colonnes ballastées accélère la consolidation grâce à un cheminement radial des eaux vers les colonnes. L'équation, établie par Barron, qui rend compte de la consolidation est :

$$
U_{r}(t)=1-\exp \left(\frac{-8 \cdot C r \cdot t}{D^{2} \cdot F(n)}\right)
$$

Avec :

$\mathrm{D}=1,03.1$ ou 1,13.1, où 1 est la distance entre colonnes respectivement pour un maillage triangulaire ou carré : $\mathrm{D}=3,39 \mathrm{~m}$,

$\mathrm{F}(\mathrm{n})=\ln (\mathrm{n})-0,75$ avec $\mathrm{n}=\mathrm{D} / \mathrm{d}=3,39$,

$\mathrm{Cr}$ est le coefficient de consolidation radial $(\mathrm{Cr}=$ $1,4.10^{-6} \mathrm{~m}^{2} / \mathrm{s}$ ),

t est le temps et Ur est le degré de consolidation,

d est le diamètre du drain $(5 \mathrm{~cm}$ pour le drain plat et $1,00 \mathrm{~m}$ pour la colonne ballastée)

Le dimensionnement du réseau de colonnes ballastées a été élaboré de façon à obtenir une consolidation Ur égale à $90 \%$ sous le bâtiment dans un délai d'environ 3 mois.

\section{4}

\section{Exécution}

\section{4}

\section{Planche d'essai}

Une planche d'essai de $400 \mathrm{~m}^{2}$ (20 m x $20 \mathrm{~m}$ ), située sensiblement à l'emplacement de la zone 2 (Fig. 5), a été réalisée au démarrage du chantier pour valider
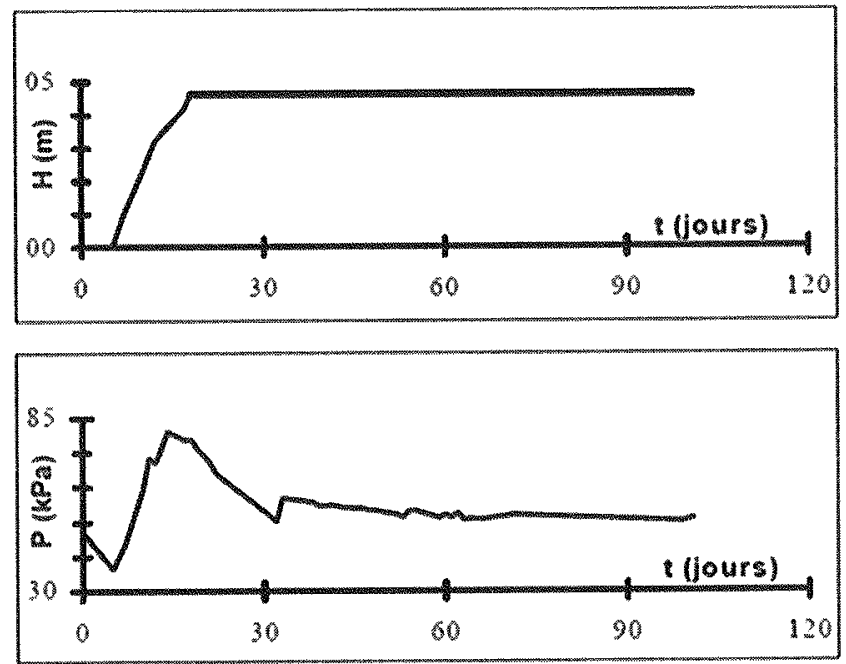

Fic $s$ Évolution de la pression interstitielle en fonction du temps et de la hauteur du remblai. 
la durée de consolidation du sol et les valeurs prévisionnelles de tassement. Un dégarnissage de colonnes ballastées en partie supérieure a mis en évidence un diamètre moyen de colonne de plus d'un mètre, confirmant ainsi l'hypothèse retenue dans le calcul de la consolidation. L'analyse des résultats des mesures de tassement et de pressions interstitielles a permis de vérifier que la consolidation s'effectuerait sur une période de 2 à 3 mois.

Les tassements mesurés lors de la planche d'essai ont permis d'extrapoler une valeur finale de l'ordre de $80 \mathrm{~cm}$ pour une hauteur de remblai de $4,50 \mathrm{~m}$. Cette valeur est proche de celle obtenue par le calcul théorique déterminé en appliquant les théories de Terzaghi (1995), Barron (1947) et Priebe $(1976,1978)$.

\section{3.}

\section{Suivi de l'instrumentation}

Le suivi du tassement a permis de constater un comportement du sol très différent d'une zone à l'autre lié entre autre aux épaisseurs de remblai mis en cuvre et à la coupe géologique (Fig. 6).



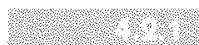

\section{Tassements absolus}

Les tassements mesurés dans les zones 1 et 2 sont de l'ordre de 0,80 à $1,10 \mathrm{~m}$ pour 4 à $5 \mathrm{~m}$ de remblai (résultat sensiblement identique à la planche d'essai). Ils varient de 0,20 à $0,40 \mathrm{~m}$ dans les zones 5 et 6 ceci pour 2 à $3 m$ de remblai.

Dans les zones centrales 3 et 4 , les piges indiquaient des tassements pouvant varier de 0,30 à $0,80 \mathrm{~m}$ pour une hauteur de remblai variable de 2 à 3 m environ (Fig. 7). Ces différents tassements sont en accord avec la coupe géologique et l'étude théorique initiale.

\section{Vences}

\section{Temps de consolidation}

En zones 1 et 2, l'analyse des courbes de tassement met en évidence un comportement différent des autres zones en ce qui concerne la vitesse d'évolution des tassements. En effet, dans les zones 5 et 6 et les zones 3 et 4 la stabilisation des tassements s'est effectuée sur une période inférieure ou égale à 3 mois. Par contre, la stabilisation complète des tassements n'était toujours pas atteinte en zone 1 et 2 après 2 à


R6. 7 Évolution des tassements en fonction de la hauteur du remblai et du temps (zones 3 et 4$)$.

3 mois de préchargement. Aussi, afin de maîtriser le planning de construction du bâtiment et en accord avec le maître d'œuvre, un remblaiement supplémentaire de $2 \mathrm{~m}$ de hauteur, en plus des $1,5 \mathrm{~m}$ déjà mis en œuvre a été programmé sur les zones 1 et 2 critiques. Ce remblaiement supplémentaire a permis de faire apparaître un supplément de tassement de $20 \mathrm{~cm}$ en l'espace d'un mois seulement.



FiG.8. Évolution du tassement après surcharge supplémentaire.

Finalement, après déchargement, une stabilisation des tassements a pu être vérifiée à partir des piges de tassements restantes et également par la mise en place de repères topographiques supplémentaires sur les poteaux du bâtiment pendant toute la durée de la construction jusqu'à réception définitive de l'ouvrage. 


\section{5}

\section{Conclusion}

En l'absence d'un substratum bien défini, la solution de traitement de sol par un maillage de colonnes ballastées sur $18 \mathrm{~m}$ de profondeur a permis la construction d'un centre commercial dans un laps de temps très court sur un terrain très compressible. Pour être bien certain de maîtriser les tassements absolus et différentiels sous les charges et en tenant compte des caractéristiques de sol sur plus de $30 \mathrm{~m}$, ce traitement a été complété par un préchargement du sol (avec un coefficient de 1,5 minimum sur la charge globale finale de l'ouvrage). Les parkings et la cour de service ont également été préchargés après mise en place de drains verticaux préfabriqués (zones $4 \mathrm{a}, 4 \mathrm{~b}, 4 \mathrm{c}, 3 \mathrm{a}, 3 \mathrm{~b}$, $3 \mathrm{c}$ de la figure 4). L'extrapolation entre le comportement à court terme (planche d'essai), et celui à long terme est resté valable en ce qui concerne les valeurs absolues de tassements sur l'ensemble du bâtiment. Par contre, en ce qui concerne les temps de consolidation, une différence importante est apparue dans les zones 1 et 2 (tassements de fluage, hétérogénéité locale ou effet de profondeur).

Ceci montre bien que les prévisions de comportement de sol sur la base de la planche d'essai (à court terme), sont nécessaires mais pas suffisantes dans le cas de sols aussi complexes et compressibles. Ceci rend nécessaire et complémentaire le suivi en temps réel de l'ouvrage. Les suivis tassométriques et piézométriques en temps réel ont permis d'ajuster les hauteurs de remblai de préchargement et les temps de consolidation afin de tenir compte à la fois des hétérogénéités très locales du sol et des charges et du phasage très particulier de préchargement par zones.

\section{Bibliographie}

Barron R.A. - Consolidation of finegrained soils by drains wells. A.S.C.E. Journal Soil Mechanics, vol. 73, SM6, 1947, p. 811-835.

Dhouib A., Stoehr B. - Techniques d'amélioration des sols par vibration profonde : origine, développement et méthodes de dimensionnement. Entretiens de la Technologie, édition euro- péenne, École centrale de Lille. Les Dossiers des technologies, Ed. Londez Conseil, 1997, p. 271-277.

Priebe H. - Die Bautechnik, vol. 53, n ${ }^{\circ} 5$ 1976, p. 160-162.

Priebe H. - Die Bautecknik, vol. 55, n 9 1978, p. 281-284.

Soyez B. - Méthodes de dimensionnement des colonnes ballastées. Bulletin du
Laboratoire Central des Ponts et Chaussées, ${ }^{\circ} 135,1985$, p. 35-32.

Terzaghi K., Peck R.B. - Soil Mechanics and Engineering Practise, John Wiley \& Sons, New York, 1948. 